\title{
DEL ANÁLISIS CRÍTICO A LA AUTORIDAD FEMENINA EN LA CIENCIA*
}

\author{
CONSUELO MIQUEO, Ma JOSÉ BARRAL MORÁN, ISABEL DELGADO ECHEVERRÍA, \\ TERESA FERNÁNDEZ-TURRADO, CARMEN MAGALLÓN \\ Universidad de Zaragoza. Grupo Genciana.
}

Partir de las vidas de las mujeres supone colocarse en un sistema de referencia que asume los supuestos epistemológicos del punto de vista feminista, elaborados y discutidos ampliamente en los últimos años. ${ }^{1}$ Para esta línea epistemológica, el conocimiento emana desde una situación social dada, en la que explícita o implícitamente está situado el sujeto conocedor. La noción de un conocimiento situado, que confronta las concepciones positivistas instaladas en la neutralidad del sujeto, ha mostrado su potencialidad teórica y práctica al posibilitar sacar a la luz e investigar problemáticas oscurecidas desde los paradigmas dominantes. Situarse en las vidas de las mujeres para construir conocimiento ${ }^{2}$ ha permitido que los estudios de género hayan abordado de manera crítica cuestiones como los hábitos de exclusión de las mujeres del saber transmitido, la denegación de autoridad epistémica femenina, la producción de

* Trabajo del Proyecto "Hechos y valores de género en la produción y difusión científica» subvencionado por el Ministerio de Ciencia y Tecnología, cofinanciación FEDER (BHA2001-2456)

1. HARSTOCK, Nancy:»The Feminist Standpoint: Developing the Ground for a Specifically Feminist Historical Materialism", en Sandra HARDING y Merrill HINTIKKA (eds.): Discovering Reality: Feminist Perspectives on Epistemology, Metaphysics, Methodology and Philosophy of Science, Dordrecht, Reidel, 1983, 283-310; HARSTOCK, Nancy: «The feminist Standpoint Revisited \& Other Essays", Boulder, Westview Press, 1998; HARDING, Sandra: The Science Question in Feminism, Ithaca, NY, Cornell University Press, 1986; HARDING, Sandra: Whose Science? Whose Knowledge? Thinking from Women's Lives. Ithaca, NY, Cornell University Press, 1991; HARDING, Sandra: "Comment on Hekman's 'Truth and Method: Feminist Standpoint Theory Revisited': Whose Standpoint Needs the Regimes of Truth and Reality?», Signs: Journal of Women in Culture and Society, 22 (1997), pp. 382-391; HARDING, Sandra: "After Absolute Neutrality», en Maralee MAYBERRI, Banu SUBRAMANIAM \& Lisa H. WEASEL (eds.): Feminist Science Studies. A New Generation. New York \& London, Routledge, 2001, pp. 291-304; HEKMAN, Susan: "Truth and Method: Feminist Standpoint Theory Revisited», Signs: Journal of Women in Culture and Society, 22 (1997), pp.341-365.

2. Ver HARDING, Sandra: Whose Science? Whose Knowledge? Thinking from Women's Lives, op. cit. 
teorías sesgadas acerca de las mujeres, la no consideración de estilos cognitivos y modos de conocimientos femeninos, la producción de teorías sobre la realidad que ocultan las actividades e intereses de las mujeres y la producción de conocimiento científico que, en lugar de servir como herramienta de cambio de las relaciones de poder, refuerza las estructuras de jerarquía y dominación ${ }^{3}$. Esta labor crítica ha ido acompañada de una construcción alternativa que ha recuperado figuras de autoridad femenina en la ciencia, así como teorías y modos de hacer que constituyen un legado que amplía, mejora y completa el cuerpo de conocimientos tecnocientíficos a nuestra disposición.

\section{PARTIR DE LAS VIDAS DE LAS MUJERES}

Según muestran las biografías de las científicas, las mujeres han estado participando en la construcción de la ciencia en todas las épocas y en todos los lugares. El deseo de conocer el mundo de un modo sistemático y colectivo, deseo que está en la base del desarrollo de la ciencia, ha llevado a muchas mujeres a desafiar estereotipos para entrar en mundos misóginos, o bien a crear espacios para la investigación científica en los lugares donde transcurrían sus vidas, originando así escenarios de libertad femenina también para la ciencia. En las últimas décadas se han publicado numerosas biografías y autobiografías de científicas de todos los tiempos, que muestran las diferentes formas en que cada una ha vivido su pasión por la ciencia; algunas de ellas han llegado al gran público y aparecen reseñadas en los libros de texto ${ }^{4}$.

La historia de las mujeres científicas saca a la luz una realidad largamente silenciada por la historia de la ciencia, en la cual las contribuciones de las mujeres permanecen, por lo general, invisibles. La misma historia de la ciencia presenta dos razones para explicar la ausencia de registro de las aportaciones de las mujeres: una, que la mayoría y los principales descubrimientos científicos han sido realizados por varones, $y$, otra, que en la investigación científica el sujeto investigador no tiene sexo, o éste no es relevante. La expresión conjunta de ambos argumentos constituye una paradoja, ya que, si aceptáramos la segunda razón, la primera no podría siquiera ser formulada. La misma contradicción sugiere que hay algo más que no se recoge en estas razones. Numerosos estudios feministas, realizados desde distintas perspectivas, han analizado las formas en que la institucionalización y la historia operan para invisibilizar a las mujeres y cancelar la autoría femenina en la ciencia, y muestran que hay ocultación cuando la historia presenta a la ciencia como un mundo del que las mujeres están ausentes ${ }^{5}$.

3. Ver HARDING, Sandra: "Comment on Hekman's 'Truth and Method: Feminist Standpoint Theory Revisited': Whose Standpoint Needs the Regimes of Truth and Reality?" y "After Absolute Neutrality", ops.cits.

4. Entre ellas cabe destacar: FOX KELLER, Evelyn: Seducida por lo vivo. Vida y obra de Bárbara MacClintock, Barcelona, Fontalba, 1983; LEVI-MONTALCINI, Rita: Elogio de la Imperfección, Barcelona, Ediciones B, 1989 (autobiografía); MAGALLÓN PORTOLÉS, Carmen: Pioneras españolas en las ciencias. Las mujeres del Instituto Nacional de Física y Química, Madrid, CSIC, 1998.

5. Entre los trabajos recientes destacamos: CABRÉ, Montserrat: "La ciencia de las mujeres en la Edad Media. Reflexiones sobre la autoría femenina», en C. SEGURA GRAIÑO (ed.): La voz del 
La condición de neutralidad, postulado positivista que niega la subjetividad del sujeto investigador, y por tanto su sexo, hace a la ciencia especialmente reacia al reconocimiento de la autoría femenina y, en general, a incluir la diferencia sexual como variable significativa. La imagen de neutralidad permite a la ciencia aparecer como garantía de verdad en el conocimiento del mundo precisamente porque se presenta como imparcial y privada de toda connotación subjetiva. Pero en la realidad los sujetos que hacen la ciencia son necesariamente hombres o mujeres, sobre quienes la supuesta indiferencia sexual tiene consecuencias distintas: en la práctica, no supone esfuerzo ni contradicción cuando el sujeto es hombre, mientras que cuando es mujer hace necesario que ésta pierda u oculte su identidad sexual. Lo mismo se refleja en el registro histórico: la condición de mujeres de las científicas quedará oculta bajo la de "neutras»; su trabajo científico, asimilado en un colectivo «neutro», no tendrá significado de experiencia femenina. La asociación implícita de lo neutro con lo masculino es perfectamente explicable en una comunidad que se autodefine como producto histórico de una sociedad masculina: el sujeto neutro, en realidad, sólo puede ser un hombre.

La imagen que presenta la ciencia tiene consecuencias importantes en el proceso de transmisión, ya que el registro histórico de las aportaciones individuales y colectivas cumple una función pedagógica. Al estudiar una ciencia, hay que aprender unos nombres que se presentan ligados a conceptos y teorías, los cuales hacen el papel de títulos: hablamos así de "leyes de Mendel» o del "principio de Arquímedes». Estos nombres pasan a formar parte de la cultura común de quienes estudian cada ciencia -y muchos de ellos a la cultura general de nuestra sociedad. La selección de nombres, que refleja el reconocimiento de autoridad otorgado por la comunidad científica de referencia, difícilmente podría incluir muchos nombres femeninos, no desde luego en la medida de su existencia e importancia, debido a los sucesivos filtros que la comunidad científica ha impuesto a las mujeres. Pero hay algo aún más significativo: en ausencia de otra información, todos los nombres ligados a descubrimientos y leyes científicas son tomados como masculinos. Es fácil comprobar esta afirmación preguntando a cualquiera que haya cursado un bachillerato o una carrera de ciencias: un gesto de sorpresa acompaña siempre a quien se entera de que alguno de los nombres aprendidos corresponde a una mujer. Por otra parte, sólo el nombre de $M m e$. Curie es citado cuando se pregunta por una mujer científica -y no hay que olvidar que recibió dos veces el Premio Nobel y que, aunque se le conozca por el apellido de su esposo, se aprende siempre precedido por el apelativo Mme. o el nombre de Marie. Informar de la identidad femenina de una científica del pasado tiene algo de transgresión, de quebrantamiento de una

silencio II, Madrid, Almudayna, 1993; ORTIZ GÓMEZ, Teresa, BECERRA CONDE, Gloria (eds.): Mujeres de ciencias. Mujer, feminismo y ciencias naturales, experimentales y tecnológicas, Granada, Universidad de Granada, 1996; HIPATÍA: Autoridad científica, autoridad femenina, Madrid, horas y Horas, 1998. 
norma. Desvelar su realidad femenina puede poner en entredicho la importancia del nombre y la de su descubrimiento, e incluso despertar sospechas sobre las verdaderas intenciones de quien proporciona la información.

En el transcurso de la investigación histórica que hemos realizado en los últimos años alrededor del tema de la determinación biológica del sexo, hemos podido comprobar la eficacia de los mecanismos de la ciencia para invisibilizar la participación de las mujeres. En el mismo principio de la investigación fuimos a dar con una científica cuyas aportaciones son muy conocidas en el campo de la biología y cuyo nombre conocíamos bien, sin sospechar que se tratara de una mujer. Como podemos leer en cualquier manual de biología, los cromosomas sexuales fueron descubiertos en 1905 por Wilson y Stevens al observar las células reproductoras de ciertos insectos ${ }^{6}$. La imagen que generalmente nos hemos formado con este dato es la de dos científicos, probablemente con barba y bata blancas, observando células en un microscopio. El nombre completo de Nettie Maria Stevens, o las imágenes fotográficas de Wilson y Stevens en sus laboratorios, producen en biólogas y biólogos actuales el efecto de romper un esquema, el cual había sido construido sin información específica sobre la identidad sexual de ambos sujetos. Vemos así que incluso aquellas mujeres que han atravesado todos los filtros de la ciencia y de su historia hasta llegar hasta nuestros libros de texto, que han conseguido tener significado en la ciencia, pierden su significado de autoridad femenina, es más: sus aportaciones pasan a engrosar el imaginario masculino bajo la supuesta condición de neutralidad.

En la mencionada investigación histórica, centrada en los primeros años del siglo XX, se han localizado otras científicas que aportaron sus descubrimientos sobre los mecanismos de determinación del sexo en diferentes seres vivos. Entre las americanas podemos citar, junto a Nettie M. Stevens (1861-1912), a Helen Dean King (1869-1955), Alice M. Boring (1883-1955), Estrella E. Carothers (1883-1957), Mary Treat, H. Randolph y Louise B. Wallace. Entre las españolas, Margarita Comas Camps (1892-1973) y Jimena Fernández de la Vega (1896-1984) ${ }^{7}$. Averiguar la condición de mujeres de los nombres encontrados en las citas se ha debido en muchos casos a la casualidad o la suerte. Según se ha comprobado, todas ellas recibieron la ratificación de la comunidad científica para sus observaciones y descubrimientos, trabajaron en los principales centros de investigación biológica del momento y publicaron en revistas prestigiosas. Y sin embargo, sacar a la luz su existencia y significación ha requerido fuerte dosis de empeño y una investigación exhaustiva en fuentes paralelas. Un dato

6. Sobre el descubrimiento de los cromosomas sexuales y el papel primordial de N. M. Stevens: DELGADO, Isabel: «Nettie Maria Stevens y la función de los cromosomas sexuales», Cronos. Cuadernos Valencianos de Historia de la Medicina y de la Ciencia, 3 (2000), pp. 239-271.

7. El papel de ambas en el contexto de las investigaciones españolas sobre la determinación del sexo es comentado en: DELGADO, Isabel. "Investigaciones biológicas españolas en torno a la determinación del sexo 1850-1936", Actas de XII Congreso Nacional de Historia de la Medicina (2002, en prensa). 
relevante es que los diccionarios biográficos al uso empiezan a recogen sus nombres en las últimas ediciones ${ }^{8}$.

A veces se ha dicho que también por parte de las científicas hay una decisión (¿una necesidad?) de ocultamiento de su identidad sexual en cuanto que pertenecientes a la comunidad científica. La profesión de científicas pide de ellas "dejar su ser mujer en la puerta del laboratorio», lo que significa también dejar fuera de su trabajo científico su experiencia del mundo como mujeres. Para ellas mismas, su valor como científicas podría verse reducido si expresaran de alguna forma su pertenencia a un colectivo femenino. Es muy posible que muchas científicas realicen o hayan realizado su trabajo plenamente imbuidas de su condición de neutralidad, y que, por tanto, no encontremos en sus obras ninguna expresión de su diferencia sexual.

En los casos de las científicas estudiadas en nuestra investigación, existen bastantes indicios de que se sentían "mujeres haciendo ciencia», y no sujetos neutros. Aunque se expresaran dentro de los límites de las convenciones del estilo científico, sus escritos muestran a menudo una colocación particular respecto del objeto de estudio -seres vivos-, y respecto de las personas que colaboraron con ellas y de las que potencialmente leerían sus observaciones. Encontramos en sus publicaciones una inusual frecuencia de agradecimientos y citas a las obras de otras mujeres, lo que puede interpretarse como expresión de un explícito reconocimiento de autoridad femenina. Así, por ejemplo, mientras en otros autores españoles es habitual citar más a Wilson en relación con los cromosomas sexuales o hablar de Stevens en masculino (Bordás, 1920; Nonídez, 1922), Jimena Fernández de la Vega escribe repetidamente el nombre de Miss Stevens y detalla sus aportaciones. De forma semejante, Nettie Maria Stevens cita a otras investigadoras de su tiempo, como Miss Boring y Miss Randolph, a quienes conoce, y a Helen Dean King, cuyas hipótesis eran opuestas a la existencia de cromosomas sexuales. Las redes de citas, a uno y otro lado del Atlántico, nos conducen hacia las mujeres fundadoras de colleges para los estudios universitarios de las mujeres en Inglaterra y Norteamérica, hacia las científicas que crearon premios de investigación y becas para que otras mujeres pudieran ampliar su formación o continuar su trabajo científico: premios como el que permitió a N. M. Stevens trabajar en 1900 en el laboratorio europeo más prestigioso, becas que llevaron a decenas de españolas a Inglaterra y Norteamérica entre 1920 y 1936 para mejorar su preparación científica. La red

8. Las biografías de Stevens y King aparecen en el suplemento publicado en 1990 del Dictionary of Scientific Biography, pero no en la edición de 1974. Ambas biografías están escritas por mujeres: MAIENSCHEIN, Jane: "Stevens, N. M.», en Charles Scribner's sons (eds.): Dictionary of Scientific Biography, New York, vol. XVIII, Suplemento II, 1990, pp. 867-869; BOGIN, Mary: «King, Helen Dean", idem., pp. 474-478. Biografías de casi todas las norteamericanas citadas pueden encontrarse en: OGILVIE, Marilyn B.: Women in Science. Antiquity through the Nineteenth Century. A Biographical dictionary with Annotated Bibliography, Cambridge-London, The M. I. T. Press, 1993. GRINSTEIN, Louise S., BIERMANN, Carol A., and ROSE, Rose K.: Women in the Biological Sciences. A Bibliography Sourcebook, London, Greenwood Press, 1997. 
internacional de mujeres universitarias que se forjó en aquellos años fue una iniciativa de mujeres que deseaban favorecer la participación de otras mujeres en la ciencia9 .

Casi un siglo después, ésta es una historia paralela de la ciencia. Una historia verdadera que pertenece tanto a la historia de la ciencia como a la historia de las mujeres. Una historia que hace de mediación necesaria para que las científicas y quienes quieren serlo puedan reconocerse en la ciencia como mujeres y dotar de significado femenino a su experiencia científica. La historia de las mujeres científicas nos proporciona una genealogía y una herencia, la herencia que nos hace dueñas como mujeres, también, del espacio de la ciencia.

\section{SESGOS ANDROCÉNTRICOS EN TEXTOS CIENTÍFICOS}

La recepción del conocimiento científico en las distintas etapas de formación produce fuertes contradicciones en las mujeres que se acercan al discurso y práctica científicos porque no se identifican bien con ese saber tan autorizado. Desde la generación de nuevo conocimiento (producción de originales) hasta los manuales universitarios, desde las monografías de síntesis hasta los titulares de los artículos de prensa diaria, este discurso se va cargando de significados de género. Para valorar el sexismo detectado es importante caracterizar la fase del ciclo científico, el tipo de fuente documental y su función social, porque el grado de estereotipia sexual del discurso científico es directamente proporcional a la cultura de género del público receptor (o reproductor). Los dos ejemplos que analizamos a continuación evidencian sesgos androcéntricos producidos por mecanismos bien distintos y de diferente repercusión.

\subsection{Manuales universitarios de neurociencia}

Los manuales universitarios son un tipo de texto científico en el que se sintetizan las teorías comúnmente aceptadas y son una buena muestra, en general, de los esquemas más arraigados en los futuros profesionales, en este caso médicos. Uno de los manuales de más amplia difusión y utilización como texto de referencia docente en las facultades de Medicina, tanto en Europa como en EEUU desde su primera edición en 1981, ha sido "Principios de Neurociencia» de Kandel, Schwartz y Jessell. De sus 4 ediciones (1981, 1985, 1991 y 2000), la cuarta destaca por un acontecimiento previo, la concesión del premio Nobel de Medicina a Eric Kandel. Este hecho marca dos diferencias de esta edición, la primera es la selección de autores de los diferentes capítulos (45 en esta edición en contraste con los 29 de la $3^{a}$ de los que solo 12 son comunes) y la segunda es la traducción a diferentes lenguas, entre ellas al español en 2001 (las otras tres ediciones no fueron traducidas)

9. Sobre las redes de apoyo de las científicas norteamericanas alrededor de 1900 ver: ROSSITER, Margaret W.: Women Scientists in America. Struggles and Strategies to 1940, Baltimore and London, The Johns Hopkins University Press, 1982. 
Hemos comparado el discurso de las dos últimas ediciones del capítulo específico de género titulado "Diferenciación sexual del sistema nervioso" (capítulo 61 en 1991 y 57 en 2000). El autor del capítulo en 1991 era el psiquiatra Dennis D. Kelly ${ }^{10}$ y en 2000 el neurobiólogo Roger A. Gorski ${ }^{11}$. Este último, autor de muchos trabajos experimentales sobre la diferenciación sexual del cerebro de las ratas que han sido y continúan siendo referencia habitual en todos los manuales de neurociencia, ya en 1971 escribía ${ }^{12}$ : «las ratas macho secretan andrógenos para masculinizar su cerebro femenino". Sus tesis tuvieron una inmediata influencia en otras ciencias y así, ese mismo año, el psicólogo Money ${ }^{13}$ afirmaba "los andrógenos dados prenatalmente producen un aumento de la inteligencia». La selección de Gorski como autor del capítulo en la edición del 2000 no parece banal sino que más bien parece obedecer a la intención de dejar definitivamente sentada (¿confirmada?) para el siglo XXI la teoría -ya común a comienzos del siglo XX y señalada en nuestro país en los años 20 por Marañón- de que "la mujer, en su evolución, es un grado intermedio entre el niño y el hombre», afirmada a finales del XX con mucho mayor peso al estar apoyada en una abrumadora sofisticación técnica.

En neurociencia, la explicación biológica de las diferencias (determinadas antes del nacimiento) entre los cerebros de hombres y mujeres ha estado y está basada androcéntricamente en el «Binomio» Cromosoma $Y \rightarrow$ Hormona Testosterona, responsable de la diferenciación cerebral llamada «masculinización» a partir de un cerebro indiferenciado, neutro o femenino. Esta teoría, de la que Gorski es uno de los máximos responsables, es mantenida en ambas ediciones, utilizando para ello diferentes recursos como una "cuidadosa selección» de evidencias y la "ocultación» o ¿el olvido? de otras (llama la atención que entre las 46 referencias de 1991 y las 40 de 2000 anteriores a 1991 sólo 5 sean comunes, que en esa red de citas no coincida ninguna referencia de los trabajos de Gorski anteriores a 1991, de los que en 1991 se citan 5 y en 2000 se citan 8 distintos y que en 1991 las citas estén actualizadas hasta ese mismo año y en 2000 sólo estén actualizadas hasta el año 1996).

Los estudios genéticos recogidos en los dos capítulos sobre una región del cromosoma Y llamada TDF (testes determining factor), les hacen afirmar en 1991 que «las gonadas en desarrollo son embriológicamente bipotenciales llegando a ser testículos si el gen TDF está presente y ovarios si no lo está» y en el año $2000^{14}$ que «sin instrucciones proporcionadas por el TDF se desarrollan ova-

10. KELLY, D.D.: «Sexual differentiation of the nervous system», en KANDEL, SCHWARTZ, JESSELL (eds): Principes of neural science, $3^{a}$ ed, Elsevier, 1991, cap. 61.

11. GORSKI, R.A.: "Diferenciación sexual del sistema nervioso", en KANDEL, SCHWARTZ, JESSELL (eds): Principios de neurociencia. $4^{a}$ ed, Mc Graw Hill, 2001, cap. 57. (edición en español de la original inglesa del año 2000)

12. SAWYER, C.H. y GORSKI, R.A.: Steroid hormones and brain function, Berkeley, University of California Press, 1971

13. MONEY, J.: «Pre-natal hormones and intelligence: a possible relationship», Impact of science on society, 21 (1971), pp. 285-290.

14. KELLY, op. cit, 1991, p. 961 y GORSKI, R.A., op. cit., 2000, p. 1132. 
rios». Pero así como en 1991 se cita el texto de Gilbert "Biología del desarrollo» ( $2^{a}$ edición de 1988), manual de referencia habitual en las ciencias biomédicas, en el que el autor afirmaba que "el desarrollo de los mamíferos es en la dirección femenina sin el efecto de los productos regulados por el cromosoma $Y »^{15}$, paradójicamente en 2000 Gorski no lo cita, ni esa edición ni la última de 2000 en la que el autor había cambiado su discurso y escribía: «en los mamíferos la determinación sexual primaria no es <por defecto $>$. La formación de ovarios y testículos son dos procesos activos dirigidos genéticamente ${ }^{16}$, describiendo a continuación la cascada de genes de los cromosomas sexuales (X e Y) y de algunos autosomas (cromosomas que poseemos ambos sexos) involucrados en esa diferenciación.

Basándose en los estudios endocrinológicos elegidos sobre los efectos de la testosterona, afirman en 1991 que «la diferenciación sexual está regulada por hormonas gonadales de la madre y de los testículos masculinos» o que «el aparato reproductor es femenino <por defecto> ya que se precisan las hormonas esteroideas para la diferenciación masculina» en 2000; no obstante, basándose en trabajos posteriores que demuestran que la testosterona es una hormona muy poco activa y que debe ser convertida en otras -en concreto debe ser aromatizada y convertida en estradiol (la hormona sexual "femenina»)- por el efecto de enzimas intermediarias para ejercer su efecto sobre el cerebro, concluyen los autores que «la principal hormona activa que determina el patrón normal de cerebro masculino en las ratas recién nacidas es el estradiol, una de las hormonas sexuales femeninas» en la edición de 1991, o que «el estradiol es la hormona masculinizante para muchas características sexualmente dimorfas», en la actual de Gorski ${ }^{17}$.

Ambos se preguntan a continuación, con retórica dialéctica, qué pasa en el cerebro femenino si el estradiol (hormona femenina) es la hormona responsable de la masculinización cerebral. La respuesta, basándose en el mismo estudio, es común: la « $\alpha$-feto-proteína». Esta es una proteína liberada por el hígado fetal que se une al estradiol circulante formando una molécula de gran tamaño que tiene mas dificultades para pasar la barrera hemato-encefálica, por lo que en las hembras llega menos estradiol al cerebro; en el caso de los machos, al circular aislada la testosterona y ser una molécula de pequeño tamaño, puede pasar fácilmente al cerebro y allí ser aromatizada a estradiol, ejerciendo así su efecto masculinizante. Se trataría pues de un problema cuantitativo y no cualitativo.

Pero Kelly, por un lado, no hace referencia a las evidencias de localización intraneuronal de la alfa-feto-proteína, a su acción como reservorio de estrógenos en el cerebro en desarrollo ni a su papel como controladora del nivel de los mismos en los cerebros de niñas y niños, tal y como se describe en los textos de fisiología desde el año 1989. Gorski, por el suyo, aunque lo apunta, y eso le

15. GILBERT, S.F.: Developmental biology $2^{\mathrm{a}}$ ed., Sinauer, 1988, p.760.

16. GILBERT, S.F.: Developmental biology $6^{\circ}$ ed., Sinauer, 2000, p.524.

17. KELLY: op. cit. p. 962 y 965 y GORSKI: op. cit. pp. 1134 y 1138, respectivamente. 
hace afirmar que "una interpretación de estos hallazgos es que: el cerebro de rata no es femenino de forma inherente, sino neutro", en el resumen final de su capítulo vuelve a escribir: "en muchos animales de laboratorio, el sistema nervioso central parece ser femenino de forma inherente, aunque puede ser necesaria una mínima exposición a los estrógenos para el desarrollo completamente normal del cerebro femenino» ${ }^{18}$. Otro olvido significativo es la identificación en 1996 de un segundo receptor estrogénico, receptor localizado en órganos genitales periféricos masculinos y en cerebro -fundamentalmente en corteza cerebral- que ha permitido asignar a esta hormona un papel en el desarrollo tanto genital como cerebral, femenino y masculino. ${ }^{19}$

Ambos capítulos, apoyados sólo en dos trabajos comunes, afirmarán que estos eventos hormonales suceden en un periodo crítico del desarrollo, que producen regiones dimórficas en el cerebro masculino, de mayor tamaño y con una mayor complejidad funcional, y que son responsables de las grandes diferencias en los comportamientos reproductivos y no reproductivos, ofreciendo diferentes ejemplos que muestran evidentes incoherencias.

Entre los comportamientos relacionados con la reproducción, basándose ambos en el mismo trabajo de 1959 sobre cobayas, incluyen las posturas coitales, la activa de "monta» de los machos, y la pasiva y receptiva denominada «lordosis» (arqueamiento de la espalda) de las hembras. Kelly, aunque considera que existen variaciones «normales» debido a la localización uterina de los numerosos fetos de la camada, especifica que hay tres tipos de hembras o machos dependiendo de su proximidad intrauterina a fetos machos (se nombran $2 \mathrm{M}$, $1 \mathrm{M}$ ó $0 \mathrm{M}$ ) y que difieren en muchas características incluyendo, en el caso de las hembras, actividad, agresividad y aceptabilidad de machos para aparearse, y en el caso de los machos, peso y tamaño de los testículos y mayor o menor dosis de testosterona para inducir agresividad. Gorski, por su parte, explica que "la observación de que los machos presentan en ocasiones lordosis y las hembras reflejo de cubrición (sic) llevó a sugerir otros mecanismos aparte del hormonal», pero concluye que «en aquella época no había pruebas suficientes sobre las diferencias sexuales estructurales dependientes de las hormonas ${ }^{20}$.

Respecto a los comportamientos no reproductivos, la síntesis es mas compleja y las conclusiones tan contradictorias que parecen haber elegido mal los datos para las conclusiones deseadas. No parece un buen ejemplo de razonamiento científico ni siquiera para quien pretenda la síntesis más ecléctica de teorías alternativas. Kelly utilizará dos casos, el primero en monos y el segundo en humanos. En Macacus rhesus, los estudios describen que las lesiones de la región órbito-frontal en crías macho producen una alteración de la discriminación espacial que no ocurre en las crías hembras, de lo que se deduce que en los machos madura antes esa región y eso hace que de adultos sean superiores

18. GORSKI, op. cit, pp. 1139-46.

19. KUIPER, G.J.M. et al.: "Cloning of ER-Beta», P.N.A.S, 93 (1996), pp: 5925-30; KUIPER, G.J.M. et al.: "Estrogen is a male and female hormone», Science/Medicine, julio-agosto (1998), pp. 36-45.

20. KELLY: op. cit., p. 968 y GORSKI, op. cit, p. 1135-6. 
en esa actividad. En el estudio en humanos se afirma que las impresiones clínicas son consistentes con la idea de que el hemisferio derecho en niñas no está especializado para una particular función cognitiva, por lo que puede retener durante más tiempo que los niños una mayor plasticidad; esta plasticidad más extensa se traduce, en el caso del lenguaje (función que se afirma es específica del hemisferio izquierdo), en que las mujeres tienen más baja incidencia de desórdenes del desarrollo asociados con la disfunción del hemisferio izquierdo, de manera que la dislexia, la afasia y el autismo infantiles son más frecuentes en hombres, siendo los deficits de lenguaje síntomas específicos de los tres síndromes. Paradójicamente pues, se podría deducir que en los monos la más temprana maduración cortical para una función deriva en una mayor precisión en la ejecución de esa función (la visión tridimensional), y en los humanos esa más temprana maduración (en este caso el hemisferio izquierdo para el lenguaje) no sólo se traduce en una mayor frecuencia de alteraciones de esa función sino también en una peor ejecución de esas funciones, ya que posteriormente afirmará que "las mujeres ejecutan mejor que los hombres todos los tests de fluidez verbal». Concluye el capítulo afirmando que «en humanos hay una considerable variabilidad en las cantidades de testosterona y estrógeno a las cuales es expuesto el feto normal», preguntándose si esas variaciones perinatales afectan el grado en el cual los comportamientos ligados al sexo son expresados más tarde en el adulto, a lo cual responde que «evidencias de esa posibilidad se han derivado de estudios en roedores» aunque a continuación escribe que «la comprensión de las diferencias sexuales en la organización neural del cerebro humano es todavía escasa», que "hay muy pocos patrones fijos de comportamiento en el repertorio humano" y que "la mayoría de los comportamientos humanos permanecen flexibles y abiertos a la modificación", en un ejemplo de «objetividad científica» sin límites ni empíricos ni lógicos.

Gorski, por su parte, utiliza los mismos síndromes clínicos (insensibilidad a los andrógenos, déficit de 5 alfa-reductasa e hiperplasia suprarrenal completa) para afirmar, por un lado, que «estos tres síndromes son compatibles con el concepto de que el aparato reproductor es femenino < por defecto> y que se precisa la acción de la hormona esteroidea para la diferenciación masculina» preguntándose a continuación: «si el programa <por defecto> de la naturaleza para el aparato reproductor es femenino ¿sucede lo mismo con el cerebro?». Unas líneas mas abajo responde que «la vía <por defecto> para los comportamientos relacionados con la reproducción también parece ser femenina» aunque, por otro lado, afirma que "hasta el momento, el estudio de estos transtornos clínicos no ha apoyado con claridad la posible importancia de las hormonas en la diferenciación sexual del comportamiento en el cerebro humano». El capítulo concluye afirmando que "los estudios realizados en animales de experimentación sugieren con claridad que el cerebro humano también sufre una diferenciación sexual durante el desarrollo inducida por las hormonas. Aunque hasta el momento las diferencias sexuales observadas en la función cognitiva de los seres humanos no parece estar relacionada con la función reproductora, los biólogos de la evolución podrán ser algún día capaces de explicar el efecto más bien amplio de 
las hormonas testiculares sobre el desarrollo del cerebro» ${ }^{21}$. Resulta difícil no diagnosticar síntomas de un residual «falocentrismo científico» que el riguroso método científico estaba destinado a filtrar, sobre todo si tenemos en cuenta la profunda revisión de esta teoría que se ha producido en las dos últimas décadas, como hemos comprobado en nuestros estudios previos. ${ }^{22}$

\subsection{Artículos de investigación sobre una enfermedad común: Alzheimer}

«(...) Existen diferencias significativas de sexo en enfermedades que afectan tanto a las mujeres como a los varones. Estas diferencias no han sido evidentes hasta ahora debido a que, en el pasado, casi todos los estudios epidemiológicos se realizaban solo en varones (...) Esta consideración debe potenciar nuestros conocimientos acerca de los mecanismos por los que la diferencia de sexo influye en la evolución y el pronóstico de determinadas enfermedades ${ }^{23}$.

Esta afirmación, que parece una conclusión de unas jornadas feministas de género y salud o de un simposium de epidemiólogos progresistas, es una de las novedades aparecidas en la extensa introdución de la decimocuarta edición del Harrison, el manual internacional más común para el estudio de la Patología Médica, que es la materia nuclear de la profesión médica. Cabe preguntarse si debemos considerarla una señal progresista o retrógrada, de signo feminista o patriarcal, dado que se trata de una realidad descubierta, que estamos construyendo, con el riesgo de esencialismo que sugieren ciertos debates feministas. El carácter esencialista que tendría la consideración de la diferencia sexual se ha utilizado como argumento filosófico de descalificación de la corriente filosófica que lo ha propuesto como objetivo político, debido precisamente a la pérdida simbólica de lo femenino que suele implicar la política igualitarista que mantiene como referente el patrón cultural y biológico masculino ${ }^{24}$.

Nosotras creemos que obviar la existencia de diferencias entre hombres y mujeres en diferentes enfermedades es una forma de sesgo de género. Ha sesgado, por un lado, gran parte de la investigación, obstaculizando un análisis y una valoración de diferencias y similitudes que permitieran una aproximación más funcional y sensata de la salud y las formas de enfermar; por otro lado,

21. GORSKI: op. cit. pp. 1134-1146.

22. BARRAL MORÁN, M.J.: "Diferencias cerebrales entre el hombre y la mujer». Área 3,4, (1996), pp. 8-15; BARRAL MORÁN, M.J. y DELGADO ECHEVERRÍA, I.: "Dimorfismos sexuales del cerebro: una revisión crítica», en: MJ BARRAL et al: Interacciones ciencia y género. Discursos y prácticas científicas de mujeres, Barcelona, Icaria, 1999, pp.129-159; BARRAL MORÁN, M.J.: "Genes, género y cultura» en MIQUEO, C. et al (eds.): Perspectivas de género en salud, Madrid, Minerva, 2001, pp. 135-162; BARRAL MORÁN, M.J.: «Sesgos de género en la difusión de las funciones del cerebro humano: estudio crítico comparado de textos anatómicos y neuroanatómicos en las universidades españolas entre los años 70-90", Actas del XII Congreso nacional de Historia de la Medicina (2002, en prensa).

23. FAUCI, A. et al: Harrison. Principios de Medicina Interna, Madrid, MacGraw-Hill, 1998, pp. 4-5.

24. Hemos desarrollado este dilema en: MIQUEO, C.:»Genealogía de los sesgos de género en la ciencia y práctica médica contemporánea", Actas del XII Congreso Nacional de Historia de la Medicina, (2002, en prensa). 
también ha perjudicado el mejor conocimiento de la realidad de esas diferencias en las formas de enfermar y de vivirse la enfermedad, en sus causas, su evolución clínica, efectos o eficacia de los tratamientos y repercusiones personales y sociales. El androcentrismo ha priorizado el uso de un modelo normativo y jerárquico sobre el que se ha construido el saber, haciendo significativa la diferencia desde la desigualdad desvalorizadora en la que han salido perjudicadas las mujeres. Porque, al menos retóricamente, la exclusión programática de las mujeres (o de las hembras en los animales de experimentación) ha estado justificada, precisamente, por esas mismas diferencias sexuales: el ciclo mensual estrogénico y la potencial maternidad, que se han considerado un riesgo añadido (y evitable) para los ensayos clínicos, y el tradicional papel doméstico y dependiente legalmente de las mujeres, por el que ellas no han sido «reclutadas» para los estudios.

El programa de investigación de la diferencia sexual propuesto en textos didácticos como el citado Harrison, es el resultado de varias décadas de estudios y de numerosa literatura de signo feminista que ha evidenciado distorsiones en tres sentidos que han sido denominadas, en general, sesgos de género ${ }^{25}$ : magnificación de diferencias donde no las hay, minimización de diferencias en otros casos y una acentuada ginecologización del saber sobre mujeres y salud. Una revisión bibliográfica realizada recientemente nos ha mostrado la emergencia de este programa, el crecimiento progresivo de la información, la aceptación de descriptores de codificación cada vez más precisos en las distintas bases de datos especializadas (Medline o Pubmed, Embase, Science Citation Index), y el desplazamiento del foco de atención de estos estudios sobre género, como se observa en la siguiente tabla ${ }^{26}$ :

Tabla $\mathbf{n}^{\circ} 1$. Distribución por quinquenios y temas de la literatura internacional circulante sobre género y salud entre 1982 y 2002

\begin{tabular}{|l|r|r|r|r|r|r|c|}
\hline \multicolumn{1}{|c|}{ Años } & $\begin{array}{c}\text { Género en } \\
\text { título }\end{array}$ & \multicolumn{1}{c|}{$\begin{array}{c}\text { Sobre } \\
\text { prejuicios }\end{array}$} & \multicolumn{1}{|c|}{$\begin{array}{c}\text { "gender } \\
\text { bias» }\end{array}$} & $\begin{array}{r}\text { Fisiopatología } \\
\text { diferencial }\end{array}$ & Método & Asistencia & $\begin{array}{c}\text { Relaciones } \\
\text { interpersonales }\end{array}$ \\
\hline $1982-1986$ & 353 & 88 & 13 & 259 & 99 & 34 & 26 \\
\hline $1987-1991$ & 932 & 185 & 35 & 948 & 608 & 140 & 84 \\
\hline $1992-1996$ & 2319 & 459 & 179 & 1512 & 1138 & 141 & 106 \\
\hline $1997-2002$ & 3251 & 560 & 186 & 1933 & 1407 & 146 & 145 \\
\hline Total & $\mathbf{6 8 5 5}$ & $\mathbf{1 2 9 2}$ & $\mathbf{4 1 3}$ & $\mathbf{4 6 5 2}$ & $\mathbf{3 2 5 2}$ & $\mathbf{4 6 1}$ & $\mathbf{3 6 1}$ \\
\hline
\end{tabular}

Fuente: Medline (elaboración propia)

25. RUIZ, T: «Igualdad de oportunidades en los servicios sanitarios: sesgos de género como determinante de la estructura de salud en la Comunidad» y VALLS, C: «El estado de la investigación en salud y género", en C. MIQUEO et al (eds.), op. cit, pp.163- 177 y 179-195; ROHLFS, I et al: «La importancia de la perspectiva de género en las encuestas de salud». Gac Sanit, 14/2 (2000), pp146-155. Una definición operativa de sesgos de género es la utilizada por Teresa Ruiz: «el planteamiento erróneo de igualdad o diferencia entre hombres y mujeres en cuanto a su naturaleza, sus comportamientos, sus razonamientos o sus necesidades; que puede generar una conducta desigual en los servicios sanitarios, incluida la investigación, y que es discriminatoria para un sexo respecto a otro» 
Nos encontramos, pues, ante un programa de investigación de superior Indice de progresividad de Laudan que la línea alternativa de la neutralidad de la diferencia sexual, porque es aplicable en un vasto campo como es la patología médica, es aplicable en todos los niveles asistenciales y por todos sus agentes o actores, y los resultados negativos son tan valiosos como los positivos ${ }^{27}$.

Decidimos estudiar la enfermedad de Alzheimer (EA) -una enfermedad común de gran trascendencia social por su alta incidencia ( $\mathrm{n}^{\circ}$ de casos nuevos al año) y prevalencia ( $n^{\circ}$ de casos existentes en un momento determinado) en sociedades muy envejecidas como las occidentales- por ser una enfermedad que afecta doblemente a las mujeres. En primer lugar porque hay un gran predominio femenino que supera su presencia en la sociedad (en España el 57,9\% de los mayores de 65 son mujeres pero suponen el 68,5\% del total de EA en grupo de edad), de tal manera que los expertos se cuestionan si ser mujer constituye un factor de riesgo para el desarrollo de la enfermedad, sin haber llegado por el momento a conclusiones claras al respecto. En segundo lugar, porque las mujeres van a padecer la EA también como cuidadoras, al ejercer el rol que habitualmente les asigna la sociedad.

La salud es un proceso complejo en el que influyen variables biológicas individuales (genéticas y hormonales, entre otras) y ambientales, entre las que destacan factores personales y socioculturales, además de los determinados por los propios sistemas de salud. El efecto de los sesgos de género puede aparecer en cualquiera de los niveles explicativos utilizados, como se refleja en la literatura internacional publicada en los últimos veinte años en 73 revistas de distintas especialidades, como Neurology (15 artículos), Alzheimer Dis Assoc Disordy Int Psychogeriatr (4 art.); Neurosci Lett, Am J Epidemiol (3 art.), utilizando sólo dos revistas de género para esta difusión: J Med Womens Assoc y J Gend Specif Med, ambas norteamericanas. ${ }^{28}$

En nuestra revisión (realizada a través de las Bases de datos Medline, Embase, Cochrane, Science Citation Index e Indice Médico Español, 1982-2002) sólo 108 trabajos contemplaban aspectos de diferencia sexual: menos de 0,3\% del total circulante sobre EA y el 9,4\% de los de epidemiología de EA. Al analizar esta literatura por temas encontramos que la mayor parte (43\%) analizan aspectos biológicos de la enfermedad (investigación básica) pues la mitad de los cuales tratan aspectos genéticos (genotipo ApoE) y un cuarto estudia los

26. Hemos seleccionando los artículos circulantes en Medline que cumplen las siguientes condiciones: a) presencia de "gender* o sex* o women* en el título del artículo; b) han sido codificados con el descriptor Mesh "prejudice» (de tipo sexista); o c) nombran explícitamente "sesgos de género» en el resumen del artículo (perfil de búsqueda: gender* near bias*)

27. MIQUEO, C. , FERNÁNDEZ-TURRADO, T. , et al.: "Progresividad del Programa género en salud. Análisis bibliométrico de la literatura internacional 1982-2002», Actas del IV Congreso Iberoamericano de Ciencia, Tecnología y Género (2002, en prensa)

28. Hemos presentado resultados parciales en diferentes congresos, cuyos resúmenes pueden consultarse en las Actas de I Reunión de metodología de la investigación en ciencias de la vida» (Zaragoza, 2001) y 18th International conference of Alzheimer's disease (Barcelona, 2002) y en la revista Neurología 17 (2002), pp. 287-288 (FERNÁNDEZ ,T. , MIQUEO C., et al) 
efectos de estrógenos y sus carencias en diferentes niveles de investigación (animal, terapia sustitutiva en mujeres con menopausias precoces quirúrgicas, efectos sobre funciones cerebrales...). En general tienden a investigar causalidad y correlación, así como líneas terapéuticas farmacológicas. En segundo lugar en frecuencia (30\%) aparecen las investigaciones de corte epidemiológico y poblacional, que pretenden describir y explicar la distribución de la enfermedad, o su ausencia, en diferentes grupos de población, y localizar los factores de riesgo asociados y los factores protectores. El tercer bloque (20\%) lo constituyen los artículos que analizan las diferencias en las manifestaciones clínica y evolutivas, métodos y técnicas diagnósticas, pautas de manejo e intervención, pronóstico y efectos de intervenciones paliativas y terapéuticas. Va emergiendo un cuarto grupo constituido por una cantidad creciente de trabajos (9\%) que analizan los efectos y repercusiones de la enfermedad en el llamado segundo enfermo, el cuidador, que habitualmente es una mujer.

Los resultados de estas investigaciones apoyan la percepción compartida entre clínicos de que atienden a más mujeres con enfermedad de Alzheimer. Los estudios epidemiológicos confirman mayor prevalencia que va aumentando con la edad, sin embargo no se confirma una mayor incidencia de EA en mujeres, salvo en edades avanzadas ( $>85$ años) ${ }^{29}$.

Los estudios genéticos y endocrinológicos que pretenden establecer una relación causal son aún menos concluyentes: ¡el genotipo XX explicaría esta diferencia en la prevalencia?, ¿de qué forma estarían implicados los estrógenos?, ¿el cese de su acción "protectora" sobre el cerebro de las mujeres, secundario a la brusca disminución que acompaña la menopausia, es un factor clave y demostrado? ¿cómo sería de eficaz la terapia estrogénica en estas mujeres con EA?, y ¿cuándo habría que aportarla, a qué mujeres, durante cuánto tiempo, con qué riesgo?, ¿es otro cofactor más que se cruza con los de edad y conductas y riesgos asociados a roles de género? Estas son las cuestiones que surgen de los trabajos analizados sin que por el momento haya datos que nos permitan dar una respuesta clara. Las únicas conclusiones aceptadas por la comunidad de expertos tienen que ver con la relación entre EA y edad avanzada, siendo difícil matizar la influencia parcial de las otras variables asociadas que se cruzan $^{30}$.

Se echan en falta estudios cuyo diseño incluya un análisis del efecto de factores ambientales y socioculturales implicados en la mayor supervivencia de personas de genotipo XX, como pueden ser las pautas de crianza y vida asociadas a roles, identidades y estereotipos de género que suelen determinar exposiciones diferenciales a los factores de riesgo, aumentando la mortalidad temprana en personas de genotipo XY. Traduce una forma de invisibilizar los efectos vinculados al factor género que los expertos aceptan implícitamente ${ }^{31}$.

29. GAO, S. et al: «The relationships betwen age, sex, and the incidence of dementia and Alzheimer disease: a meta-analysis», Arch Gen Psychiatry, 55 (1998), pp. 809-15.

30. BARRET, A.M: «Probable Alzheimer's disease: gender-related issues», J Gend-Specif-Med. JanFeb; 2/1(1999), pp. 55-60.

31. PRENCIPE, M. et al.: "Prevelence of dementia in an elderly rural population effects of age, sex, and education", J Neurol Neurosurg Psychiatry, 60 (1996), pp. 628-33. 
Las teorías patogénicas actuales del curso evolutivo de la EA comienzan a hablar del concepto de "reserva cognitiva» como un factor protector en su evolución, que daría lugar a formas más benignas de la enfermedad. Se aceptan la «deprivación cognitiva cultural» de las mujeres (menor escolarización generalmente asociada a roles de cuidado), y la deprivación económica que afecta a una gran parte de la población femenina (carente de recursos propios que empeora al envejecer, con pérdidas del poder adquisitivo en separaciones y viudedad que determina disminución del nivel de autonomía) como hipótesis explicativas de la mayor prevalencia de la enfermedad. Son necesarios estudios que analicen estos efectos que repercuten en la enfermedad de Alzheimer, cuestionen los instrumentos de medida utilizados y originen un perfil morboso diferente en hombres y mujeres que sea independiente de factores biogenéticos, con el objeto de conseguir políticas igualitarias de distribución de recursos y de intervenciones socioeducativas preventivas.

También hay carencia de investigaciones que analicen otros factores que tienen que ver con el diferente modo de envejecer de hombres y mujeres. Envejecer añade sobrecargas y estrés a las mujeres, lo que parece estar relacionado con mayores exigencias funcionales a nivel cerebral y mayor riesgo. Las mujeres tienen cargas y responsabilidades cotidianas hasta edades muy avanzadas, son cuidadoras hasta el mismo momento en que pasan a ser cuidadas, lo que hasta cierto nivel puede ser un factor protector; pero cuando estas demandas son excesivas, se convierten en factor de riesgo; mientras que en el caso de los hombres, estas dificultades cognitivas puede pasar desapercibidas durante más tiempo por carecer ellos de responsabilidades ${ }^{32}$.

Otro aspecto que evidencia efectos de sesgos de género tiene que ver con la aparición creciente de trabajos que inciden en el perfil femenino de la enfermedad, tipo "Mujer y Enfermedad de Alzheimer», siendo raras (en nuestra muestra hemos encontrado un caso) las descripciones específicas de la «enfermedad en el hombre». Llama la atención esta actitud que contradice lo que es habitual en la descripción médica (describir en función de lo más frecuente) y además opera de un modo perverso al ignorar aspectos diferenciales más significativos como los que hemos ido mencionando.

La experiencia clínica no encuentra diferencias esenciales en las manifestaciones conductuales de la enfermedad, y los hallazgos en las pruebas de rendimiento cognitivo pueden explicarse por aspectos mencionados en relación a exposición diferente a tareas escolares que sesgan la evaluación en función de los instrumentos con los que habitualmente se mide el deterioro cognitivo ${ }^{33}$. Son necesarios nuevos diseños de investigación con instrumentos libres de sesgos para obtener datos más concluyentes en cada uno de los niveles analizados.

32. BOADA, M. y col: "Coste de los recursos sanitarios de los pacientes en riesgo ambulatorio diagnosticados de E de Alzheimer en España", Med Clin (Bar), 113 (1999), pp. 660-95.

33. SCHMAND, B. et al : "Relation betwen education and dementia: the roles of test bias revisited», J Neurol Neurosurg Psychiatry, 59 (1995), pp. 170-4. 
Sorprende que en la revisión más reciente publicada en España ${ }^{34}$, que analiza trabajos similares a los de la nuestra, los autores hagan una reflexión explícita sobre la importancia de identificar e intervenir en factores de riesgo vinculados a conductas de género (que reducen al efecto del analfabetismo), incidiendo en las situaciones de desigualdad social como generadoras de "enfermedad añadida» en la mujer.

\section{3. ¿QUÉ CAMBIOS HA PRODUCIDO EL FEMINISMO EN LA CIENCIA ESPAÑOLA?}

El feminismo académico de nuestro país se ha interesado por la ciencia más tarde y mucho menos que sobre cualquier otra actividad social o campo del saber, como se desprende de la exhaustiva información proporcionada por el Libro Blanco de los estudios de las mujeres de las universidades españolas (1975-1990) y su Actualización, correspondiente al quinquenio 1992-1996. Los datos cuantitativos de docencia (23), investigación (5), publicaciones (4) y actividades de divulgación (34) en la materia de ciencia naturales no alcanzaban este quinquenio el $1 \%$ de la numerosa actividad desarrollada por estos estudios en España (66/6.959), aunque esas 66 actividades duplicaban el total de las aportaciones realizadas en los 15 años anteriores. Respecto a los temas y resultados obtenidos pueden distinguirse tres líneas, de acuerdo con la revisión de Teresa Ortiz: 1) los que analizan el discurso de la ciencia y el papel que juegan símbolos y metáforas y sesgos sexistas en su construcción; 2) los que se centran en las descripciones científico-médicas de la naturaleza femenina y de las diferencias sexuales, y 3) los que estudian la organización social de la práctica científica, siendo este campo el que goza de mayor aceptación en la propia comunidad científica $^{35}$. La sucesión de libros colectivos de género y ciencia que se han publicado desde el primer monográfico de 1993 de Arbor (editado por Eulalia Pérez Sedeño), muestra la especificación y cambio del foco de atención temática, y el progresivo crecimiento de la pequeña comunidad de investigadoras o estudiosas en torno a los tres núcleos universitarios más activos o constantes del país -Granada, Madrid y Zaragoza-, así como la irrupción de diversos núcleos médicos organizados en torno a la Red de Médicas y Profesionales Sanitarias existente desde 1998 (coordinada desde el CAPS por la internista catalana Carme Valls) o promovidos por la propia Sociedad Española de Epidemiología y Administración Sanitaria (SESPAS). ${ }^{36}$

34. ALBERCA, R. y MONTES-LATORRE, E: «Enfermedad de Alzheimer y mujer», Rev Neurología, 35. $/ 6$ (2002), pp. 571-579.

35. ORTIZ, T. , MARTÍNEZ, C. , SEGURA, C. et al.: Universidad y feminismo II. Situación de los estudios de las mujeres en los años 90, Granada, Universidad de Granada, Feminae, 1999; ORTIZ, T.: "Feminismo, ciencias naturales biomédicas: debates, encuentros, desencuentros», en: Cambiando el conocimiento: universidad, sociedad y feminismo, Oviedo, KRK, 1999, pp. 223-245.

36. Sin contar la importante labor de difusión de las traducciones, los libros colectivos publicados han sido: PÉREZ SEDEÑO, E. (comp.): Mujer y ciencia. Arbor CXLIV, (1993) n565, (monográfico); 


\section{La situación de las mujeres en el sistema ciencia y tecnología}

El conjunto de los estudios sobre la situación de las mujeres en el sistema de ciencia y tecnología pone de manifiesto ciertas paradojas. La principal es la que señala que la participación de las mujeres ha crecido en números absolutos de un modo importante, aunque al mismo tiempo se ha estancado su acceso a los puestos de responsabilidad más altos, tanto en el sector privado de trabajo (empresas) como en las instancias públicas, instituciones y medio académico. Ya en el año 1996-97 las mujeres alcanzaron una representación paritaria en el acceso a la universidad: el 52,4\% de los estudiantes universitarios eran mujeres, de las que la mayoría cursaban diplomaturas (67\%), y de éstas un $24 \%$ estudios técnicos (ingenierías o arquitectura); entre los becarios la representación era de un $44 \%$ de mujeres frente al $66 \%$ de hombres, aumentando la diferencia entre los doctores (30\% y 70\%). En el ámbito profesional académico, se observa la concentración de mujeres en los puestos inferiores del personal docente e investigador (PDI): 43,8\% de Profesoras Ayudantes, 30\% de Titulares, 10,8\% de Profesoras Eméritas y $10 \%$ de Catedráticas. Pero alarma la situación externa al ámbito académico pues tan sólo un 3,6\% de la masa activa femenina trabaja en investigación científica y/o tecnológica, tanto en ámbitos privados como públicos. La pirámide de la población femenina en el principal centro investigador del país CSIC (Centro Superior de Investigaciones Científicas) traduce esta anomalía, aunque ha cambiado en las últimas décadas, como se desprende de la comparación entre 1971 y 2001 por categorías: Colaboradoras de Investigación: 21\% a 38\%; Investigadoras: $25 \%$ a $26 \%$ y Profesoras de Investigación: del 8\% al 13\%. La distribución etaria del personal refleja con mayor fidelidad el retraso en el acceso y la falta crónica de promoción pues muchas mujeres llegan a edades próximas a la jubilación sin haber dejado de ser Colaboradoras o Titulares, mientras que son raros los casos de varones que no han pasado, al menos, a Investigadores ${ }^{37}$.

ORTIZ GÓMEZ, T., BECERRA CONDE, G. (eds.): Mujeres de ciencias. Mujer, feminismo y ciencias naturales, experimentales y tecnológicas, Granada, Universidad de Granada, Instituto de Estudios de la Mujer, 1996; PORTAL NIETO, A.M. (ed.): Mujeres: Ciencia, tecnología y medio ambiente, Castellón de la Plana, Universitat Jaume I, 1998; BARRAL, M.J., MAGALLÓN, C. , MIQUEO, C. , SÁNCHEZ, D. (eds): Interacciones ciencia y genero. Discursos y prácticas científicas de las mujeres, Barcelona, Icaria, 1999; CRUZ RODRÍGUEZ, M. , RUIZ HIGUERAS, L. (eds.): Mujer y ciencia, Jaén, Universidad de Jaén, 1999; PÉREZ SEDEÑO, E.; ALCALÁ CORTIJO, P.: Ciencia y género, Madrid, Facultad de Filosofía-Universidad Complutense, 2001; BERNIS CARRO, C. et al.: Salud y género: la salud de la mujer en el umbral del siglo XXI. XIII. Jornadas de Investigación Interdisciplinaria sobre la Mujer, Madrid, Universidad Autónoma de Madrid, Instituto Universitario de Estudios de la Mujer, 1999; MIQUEO, C. , TOMÁS, C.. , TEJERO, C. , BARRAL, M.J. , FERNÁNDEZ, T. , YAGO, T. (eds): Perspectivas de género en salud. Fundamentos científicos y socioprofesionales de diferencias sexuales no previstas, Madrid, Minerva, 2001; PÉREZ SEDEÑO, E.(ed.): Las mujeres en el sistema de ciencia y tecnología. Estudio de casos, Madrid, Organización de Estados Iberoamericanos (OEI), 2001; FERNÁNDEZ VARGAS, V. , SANTESMASES, M.J. (eds.): Ciencia y tecnología en el CSIC: una visión de género, Arbor 2002, CLXXII, n 679-680 (monográfico).

37. GARCÍA DE LEÓN, M.A., GARCÍA DE CORTÁZAR, M.: Las académicas:profesorado universitario y género, Madrid, Instituto de la Mujer, 2001; SANTAMARÍA, C.: "Las mujeres españolas ante el 
La segunda de las contradicciones es la discriminación horizontal, espacial o territorial, es decir, la segregación de hombres y mujeres en ciertas especialidades, campos o áreas de trabajo. En el Centro Superior de Investigaciones Científicas (CSIC) donde hay un 30,9\% de científicas en total (658 mujeres y 1470 hombres), la distribución general por áreas oscila entre Ciencia y Tecnología Físicas $(8,90 \%)$ y Ciencia y Tecnología de los alimentos (39,8\%). En las menos femininizadas se observan, a su vez, espacios casi ausentes de mujeres como el Instituto de Optica (3 de 18) o el Instituto de Matemáticas y Física Fundamental (4 de 25) ${ }^{38}$. Y en la élite de esa institución, la categoría de Profesores de Investigación, esta segregación territorial es más elocuente, oscilando entre el 28\% en Ciencia y Tecnología de Alimentos, hasta el 3\% de Ciencia y Tecnología de Materiales o el 7\% Ciencia y Tecnología Químicas; en Biología y Biomedicina las mujeres ocupan en la actualidad el 13\% de los cargos directivos, pero la situación es deseperanzadora porque en este periodo de cambios sociales evidentes para las mujeres ha descendido la proporción de investigadoras (del $41 \%$ al $23 \%$ entre 1971 y 2001, difícilmente comprensible dado el aumento de la proporción de entrada) La revisión de los sistemas de promoción profesional ha revelado que no ha cambiado la proporción por sexos del Comité Científico Asesor de 1993, que los ocho coordinadores de área son hombres, que solo hay una mujer entre los seis cargos asignados, que en la promoción a Profesores de los años 1999 y 2000 las posibilidades fueron de 1:37 y de 1:11 para un investigadora y para un investigador. Las evaluadoras han insistido en el interés de los análisis de estos procesos de evaluación interpares y han propuesto a la dirección del CSIC un nuevo sistema de composición de los tribunales que asuma la paridad de género, con el fin de evitar los efectos negativos de un sistema en espiral o autoalimentado que parece favorable para los hombres ${ }^{39}$

¿Es esa falta de reconocimiento de la actividad y excelencia femenina una situación propia de países latinos de tradición católica o es un mal endémico de la Europa comunitaria? El mayor estudio realizado en nuestro entorno europeo es el conocido Informe Etan 2000, en el que se definía la situación de «antieconómica e injusta, un anacronismo, se añadía, porque el abuso del "círculo de amigos", aunque ha cambiado en las últimas décadas, es contrario a la ciencia

conocimiento científico y tecnológico», en E. PÉREZ SEDEÑO, op. cit., 2001, pp. 41-60; Informe ETAN (vide infra); ALCALÁ, P.: "Españolas en el CSIC», en ORTIZ, T. et al, op. cit., 1996, pp. 61-75; FERNÁNDEZ VARGAS, V., SANTESMASES, M.J. (eds.), op. cit., 2002.

38. MOYA DE GUERRA, E.: "Mujeres en ciencia y tecnologías físicas en el CSIC», Arbor 679-680 (2002), pp. 562-576.

39. DE PABLO, F.: «Biología y biomedicina: un área de mujeres fértiles», Arbor 679-680 (2002), pp. 577-596. En Europa se ha realizado más de una veintena de estudios sobre los procesos de evaluación interpares desde el inicial de WENNERAS, C. , WOLD, A.: «Nepotism and sexism in peer review», Nature 387 (1997), pp. 341-343, que determinó cambios significativos en el consejo nacional de investigación médica sueco. Se han obtenido interesantes datos sobre diferencias de género, estilos de trabajo y publicación, composición sesgada de comités de evaluación, proporción de éxitos, etc., no hallándose, sin embargo, diferencias estadísticamente signifitivas o evidencias de nepotismo como en aquél primero. [Informe Etan, v.infra] pp. 33-45. 
y además niega a muchas mujeres, para ofrecérselas a los hombres, los beneficios de una carrera científica tales como la satisfacción de la propia curiosidad, la posibilidad de establecer prioridades individuales, un estatus determinado y un cierto grado de autonomía ${ }^{40}$. Y en el ámbito latinoamericano la situación es similar: en Uruguay el $23 \%$ de los puestos de director de investigación están ocupados por mujeres y en Argentina ocupan el 9,6\% de los puestos superiores de la CONICET, alcanzando el nivel superior de la carrera el 0,4\% de las mujeres frente al 4,5\% de los hombres ${ }^{41}$.

\section{Conciencia y propuestas de cambio}

Nunca las mujeres han estado tan preparadas para acceder al conocimiento y la investigación en estas áreas, pero su situación de discriminación tanto jerárquica como territorial es alarmante y puede considerarse un derroche social. Pero, sabiéndolo ¿se han articulado medidas correctoras o es un «conflicto

40. Comisión Europea, Dirección General de Investigación-Política científica de la Unión Europea, Promover la excelencia mediante la integración de la igualdad entre géneros: Informe del Grupo de trabajo de ETAN sobre las mujeres y la ciencia, Luxemburgo: Oficina de Publicaciones Oficiales de las Comunidades Europeas, 2001, 157 págs. (p. 5). Este informe ha revelado que en la industria, el porcentaje de mujeres directoras/presidentas de empresas que participan en proyectos de investigación es del 4\% en España y un 6\% en Europa, observándose diferencias considerables. Por ejemplo, en Alemania, de las 57 grandes empresas analizadas, sólo 10 tenían directivas mujeres en 1996 (1,2\%), contabilizándose 28 cargos superiores, 65 cargos medios y 107 cargos inferiores, oscilando esta distribución entre las más masculinas como Audi (0/0/23) o Wolkswagen (0/3/6) y las más feminizadas como Philips o Hewlett-Packard (5/12/2) y Unilever (7/10/14) (p. 139). Otro indicador de reconocimiento de autoridad femenina es su aceptación en las Academias, las instituciones más elitistas. Sólo en 12 de las 43 nacionales analizadas, las mujeres han superado el $5 \%$ de sus miembros (las más antiguas y conocidas como la francesa, británica o alemana no pasan del 3,6-4\%), formando parte de sus consejos directivos en proporciones visibles en los casos de la alemana (4/46), irlandesa (3/21), danesa (1/8), británica (2/21), estadounidense (3/17) y canadiense (4/18) (cfr. Etan pp. 141-142). Pero los datos de instituciones públicas, generalmente más permeables al acceso de las mujeres, no justifican el optimismo. En torno al 5\% de los rectores de Francia, Alemania o Gran Bretaña son mujeres, superando esa cota Suecia (18\%) mientras que el techo de catedráticas no pasa del 13\%. El desequilibrio en los centros de investigación muestra la misma imagen piramidal, llamando la atención las diferencias por áreas científicas. En el CERN (Centro Europeo para la Investigación Nuclear) son mujeres el $5 \%$ de los jefes de equipo, el $7 \%$ de los jefes de equipos adjuntos y el $10 \%$ de los jefes de sección. En el CNR (Centro Nazionale de la Ricerca) de Italia, el 6\% de los directores de institutos, centros de estudios y grupos nacionales son mujeres. Mientras que constituyen el $12 \%$ de los jefes de equipo del EMBL de Leipzip (Instituto Europeo de Biología Molecular) y del Laboratorio de Biología Molecular del Consejo de Investigación Médica (MRC), el 20\% del Instituto de Inmunología de Basilea o del Fondo Imperial para la Investigación del Cáncer de Londres, el 23,9\% del Instituto Pasteur de París; mientras que en tres institutos de investigación en ciencias de la vida creados recientemente en Portugal, el $45,2 \%$ de los jefes de equipo de investigación son mujeres.

41. [Informe ETAN] op cit. pp. 7-19, 141-142; KOCHEN, S; FRANCHI, A; MAFFÍA, D,: «La situación de las mujeres el sector científico-tecnológico en América latina. Principales indicadores de género", en E. PÉREZ SEDEÑO (2001), op. cit., pp.19-39; BIELLI, A; BUTI, A; VISCARDI, N.: «Participación de mujeres en investigación científica de nivel universitario en Uruguay», en Idem, pp. 93-123. 
clandestino» del que ni siquiera tienen conciencia quienes lo viven y menos aún quienes lo padecen o padecerán?

En nuestro país, el estudio de Cristina Santamaría ${ }^{42}$ ha evidenciado que las estudiantes carecen de un visión global del mundo de la tecnociencia, pero sobre todo desconocen la situación de las mujeres que trabajan en ellos. Resulta ilustrativo que no sepan valorar si en sus universidades respectivas hay más mujeres o varones catedráticos, o si sus autoridades máximas en facultades y en la misma universidad son mujeres o varones. El conocimiento y reconocimiento de esta realidad quedarían, pues, relegados a la fase de inserción al mercado de trabajo, y esta situación se producirá desde una concepción atomizada, individualista y competitiva que en nada ayuda a resolver el problema. El profesorado universitario tiene una clara conciencia de las situaciones de discriminación de las mujeres en CyT, conciencia que si bien es más acusada en ellas, no es ajena a las manifestaciones y posiciones que asumen los profesores varones. Son las investigadoras extracadémicas las que presentan un mayor grado de conocimiento (experiencial fundamentalmente) de la situación de las mujeres ante el sistema CyT: reconocen en los condicionantes de género (heteronomia femenina, doble jornada, trabajo invisible, responsabilidades familiares y domésticas, etc.) el verdadero "muro» para el reconocimiento, el prestigio y autorización de las mujeres en el quehacer científico o tecnológico. Por ejemplo, el 54,3\% de las investigadoras entrevistadas considera que en España existen muy pocas mujeres trabajando en estos campos, y el $64,7 \%$ opina que una mujer tiene más obstáculos que un varón para dedicarse a estas disciplinas, porcentaje que aumenta a un $74 \%$ entre quienes desarrollan su labor en las empresas privadas, alcanzando el $90 \%$ cuando se les solicitaba caracterizar el tipo de obstáculo: las obligaciones familiares. Respecto a la distribución de capacidades y prestigio, también entre ellas se afirma lo que ha resultado una constante en todos los niveles educativos: que lo propio de las mujeres es la capacidad intuitiva necesaria para la imaginación científica y tecnológica, mientras que lo característico de los varones es la disponibilidad para viajar, y que las profesiones más prestigiosas son medicina, ingenierías e investigación científica, observándose que no existe, tampoco en este colectivo, un discurso único y articulado sobre el mecanismo que produce esta situación o la estrategia de cambio. Respecto a la conciencia de las investigadoras experimentadas acerca de este problema, la respuesta de las entrevistadas para los informes de área del CSIC reveló escotomas (puntos ciegos) de su conciencia relacionados con la edad (y la carrera profesional), de modo que parece repetirse lo que ya ha sido denunciado hasta en Nature ${ }^{43}$ : que "cada generación de mujeres empezaba creyendo que la dis-

42. SANTAMARÍA C., op. cit. pp. 41-60. Basado en encuestas dirigidas a estudiantes y profesores de enseñanzas medias y universitarios e investigadoras, resulta especialmente interesante por el análisis -y novedosa tipificación- de los «discursos» hallados en cada una de las poblaciones estudiadas.

43. Nature, 401 (1999), p. 99. 
criminación por razón de género se «resolvió» en la generación anterior y que no les afectaba» ${ }^{44}$.

No obstante el problema de la escasa conciencia de la situación, se están articulando medidas de "resolución». En el conjunto de iniciativas habidas, nos parecen significativas las cinco propuestas del informe de 2002 del área menos feminizada en el CSIC, Ciencia y tecnología de ciencias físicas: 1) realización de un estudio estadístico y retrospectivo de la situación de la mujeres en el CSIC que incluya tanto datos profesionales como personales; 2) elaboración sistemática de estadísticas desglosadas por género (de solicitudes/concesiones de becas, contrataciones, acceso a escalas superiores y participación en tribunales, etc); 3) política activa para asegurar mayor y apropiada participación de mujeres en los foros de toma de decisiones y de política científica: órganos directivos, comisiones de selección, tribunales, comités de expertos, representaciones institucionales, etc; 4) programa de apoyo a la maternidad de modo que no perjudique la carrera profesional de las mujeres porque «la organización actual del trabajo científico se ajusta a modelos de trabajo masculinos», con medidas como creación de guarderías, flexibilización de los límites de edad o tiempo en las convocatorias de becas, contratos postdoctorales, etc.; 5) políticas de visibilidad y motivación dirigidas a todo el sistema educativo con el fin de difundir las aportaciones de las mujeres en este campo y modificar los estereotipos sexuales.

También es significativo que, de acuerdo con su estilo de trabajo diario, en el resquicio que las científicas dejan en sus informes para la expresión de lo subjetivo - de valores morales o políticos no fundamentables en datos de observación cuantificados-, la autora de este informe, la física y profesora del CSIC Elvira Moya de Guerra, eligiera la cita de un artículo de la prensa diaria (El País, Vicente Verdú) para enunciar el sentido o dirección de los cambios propuestos: "tras la masculinización femenina emerge una feminización de la virilidad», "antes había que llegar a ser alguien, ahora se trata de llegar a ser YO». Y que terminara poniendo en cuestión -con el riesgo que ello representa en la comunidad científica, y como hicieron otras de la misma institución en sus respectivos informes- la máxima del "éxito a cualquier precio». ${ }^{45}$

44. Sobre el tema en países latinoamericanos son útiles varios capítulos del libro editado por PÉREZ SEDEÑO, op. cit., 2001, y ALMODÓVAR, M.: «Mujer y ciencia en Iberoamérica. Invisibilidad y familia» en ORTIZ, T. , op . cit. pp. 83-95; y en España: GARCÍA DE CORTÁZAR, M., GARCÍA DE LEÓN, M.A., op. cit; GARCÍA DE LEÓN, M.A. : Herederas y heridas. Sobre las élites profesionales femeninas, Madrid, Cátedra-Feminismos, 2002, además de los estudios ya citados sobre CSIC de Arbor de agosto de 2002. También nosotras contribuimos a esta memoria en unas jornadas: MIQUEO, C.: "Contrastar experiencias: diversidad de modelos para las científicas. Una mesa redonda", en: BARRAL, M. J. et al (199), op. cit., pp. 291-324.

45. MOYA DE GUERRA, E.: op. cit. p. 573-575. Las recomendaciones para la comunidad europea pueden verse en pp. 81-95 del citado informe ETAN (www.cordis.lu/rtd2002/science-society/ women.htm) 